\title{
Danshen (Salvia miltiorrhiza) Compounds Improve the Biochemical Indices of the Patients with Coronary Heart Disease
}

\author{
Boyan Liu, ${ }^{1}$ Yanhui Du, ${ }^{1}$ Lixin Cong, ${ }^{1}$ Xiaoying Jia, ${ }^{2}$ and Ge Yang $^{1}$ \\ ${ }^{1}$ Department of Geriatrics, Affiliated Hospital, Changchun University of Traditional Chinese Medicine, Changchun 130000, China \\ ${ }^{2}$ Department of Neurology, Jilin Province People's Hospital, Changchun 130000, China \\ Correspondence should be addressed to Ge Yang; yangge338@163.com
}

Received 25 February 2016; Revised 16 April 2016; Accepted 3 May 2016

Academic Editor: Roja Rahimi

Copyright (C) 2016 Boyan Liu et al. This is an open access article distributed under the Creative Commons Attribution License, which permits unrestricted use, distribution, and reproduction in any medium, provided the original work is properly cited.

Danshen was able to reduce the risk of the patients with coronary heart disease (CHD), but the mechanism is still widely unknown. Biochemical indices (lipid profile, markers of renal and liver function, and homocysteine (Hcy)) are closely associated with CHD risk. We aimed to investigate whether the medicine reduces $\mathrm{CHD}$ risk by improving these biochemical indices. The patients received 10 Danshen pills $(27 \mathrm{mg} / \mathrm{pill})$ in Dashen group, while the control patients received placebo pills, three times daily. The duration of follow-up was three months. The serum biochemical indices were measured, including lipid profiles (LDL cholesterol (LDL-C), HDL-C, total cholesterol (TC), triglycerides (TG), apolipoprotein (Apo) A, ApoB, ApoE, and lipoprotein (a) (Lp(a))); markers of liver function (gamma-glutamyl transpeptidase (GGT), total bilirubin (TBil), indirect bilirubin (IBil), and direct bilirubin (DBil)); marker of renal function (uric acid (UA)) and Hcy. After three-month follow-up, Danshen treatment reduced the levels of TG, TC, LDL-C, Lp(a), GGT, DBil, UA, and Hcy $(P<0.05)$. In contrast, the treatment increased the levels of HDL-C, ApoA, ApoB, ApoE, TBil, and IBil $(P<0.05)$. Conclusion. Danshen can reduce the CHD risk by improving the biochemical indices of CHD patients.

\section{Introduction}

Coronary heart disease (CHD) is the leading cause of death in the world $[1,2]$. The number of CHD patients will reach 82 million in 2020 [2]. CHD still cannot be cured and present treatment prevents symptom development and reduces the incidences of heart attacks. CHD therapy mainly includes exercise-based cardiac rehabilitation [3], the changes of the dietary patterns (stopping alcohol consumption) [4], and medication [5] as well as aortic valve replacement and coronary-artery bypass graft surgery [6]. Therefore, due to the lack of effective therapy, it is necessary to discover new treatments for preventing CHD risk.

Traditional Chinese medicine (TCM) has a profound history and has been practiced in many diseases. It is an approach to exploring new medicine and mechanism for CHD therapy [7]. Danshen (Salvia miltiorrhiza), a form of TCM, is often applied in the therapy for coronary heart disease $[8,9]$. The results of a number of publications pointed to antioxidant [10, 11], anti-inflammatory [12], protective [13], or antiplatelet [14] properties of Danshen and its active compounds. A salvianolic acid B ( $\mathrm{SaB})$, an important bioactive ingredient in the root of Danshen, is being suggested to be responsible for its antioxidant property [10]. Other active water-soluble compounds, such as protocatechuic aldehyde (PAl), 3,4-dihydroxyphenyl lactic acid (DLA), and SaB with peroxides scavenging activities, were able to prevent the expression of adhesion molecules in vascular endothelium and inhibit vascular damage and the components such as tanshinone IIA and tanshinone IIB can inhibit the activity of NADPH oxidase and the aggregation of platelet [11]. This may explain the medicine usage for treating various microcirculatory disturbances. Anti-inflammatory properties of major ingredients $\mathrm{SaB}$, tanshinone IIA (Tansh), and protocatechuic acid preventing the expression of adhesive molecules, cytokines, chemokines, and platelet P-selectin were also observed [12]. Furthermore, low-concentration Danshen was able to protect human umbilical vein endothelial cells 
(HUVECs) and improve their functions [13]. Its main components, rosmarinic acid, lithospermic acid, SaB, salvianolic acid $\mathrm{C}(\mathrm{SaC}), \mathrm{D}(\mathrm{SaD})$, and and $\mathrm{H} / \mathrm{I}(\mathrm{SaHI})$, have also antiplatelet potential [14].

It is well known that the changes in the levels of a number of biochemical parameters are directly or indirectly associated with the risk of occurrence of CHD. Firstly, low-density lipoprotein cholesterol (LDL-C) is an important risk factor for $\mathrm{CHD}$ and the concentration should be well controlled for reducing the incidences of $\mathrm{CHD}$ [15], while the concentration of high-density lipoprotein cholesterol (HDL-C) is strongly and inversely associated with CHD risk [16]. A correlation with the occurrence with this disease was also observed with the changes in levels of total cholesterol (TC) and triglycerides (TG) $[17,18]$ as well as in the case of apolipoproteins A (ApoA), B (ApoB), E (ApoE) and lipoprotein (a) $(\mathrm{Lp}(\mathrm{a}))$ genes expression profile changes [19-22]. The markers of liver function such as $\gamma$-glutamyl transpeptidase (GGT) [23], total bilirubin (TBil) [24], indirect bilirubin (IBil), and direct bilirubin (DBil) [25] are also related to CHD risk. Furthermore, serum level of uric acid, one of markers of renal function [26, 27], can also reflect the severity of CHD [28]. Moreover, the high concentration of homocysteine (Hcy) concentration is regarded as a risk factor for cardiovascular disease $[29,30]$.

Several clinical trials showed also positive effects in the field of above-mentioned parameters, including improvement of the lipid patterns of hyperlipidemic patients [31] and protective properties in the patients with liver [32] or renal injury [33]. We hypothesized that Danshen may be able to reduce the incidences of $\mathrm{CHD}$ by improving these biochemical indices (lipid profile, markers of renal and liver function, and Hcy) of CHD patients. Therefore, placebo-controlled, prospective, and randomized study was conducted to investigate the effects of the medicine on biochemical indices of CHD patients and explore the possible mechanisms of its functions.

\section{Methods}

2.1. Patients. Before the study, all protocols were approved by the human ethical committee of Affiliated Hospital of Changchun University of Traditional Chinese Medicine. The study was conducted according to the principles of the Declaration of Helsinki [34]. All patients signed the informed consents before being enrolled in this study. From March 2011 to June 2012, 432 CHD patients attended our hospital. A total of 126 patients met following inclusion criteria and were considered for enrollment in the study.

2.2. Inclusion Criteria. Inclusion criteria were given according to guidelines for the management of patients with myocardial infarction [35-37]. All patients should have one of the following clinical symptoms: (1) unstable angina; (2) STelevation myocardial infarction (STEMI) and non-STEMI; (3) patients undergoing coronary-artery bypass grafting (CABG) surgery; (4) patients undergoing undergone percutaneous coronary intervention (PCI); patients undergoing coronary-artery stent; (5) CHD determined by angiography.
2.3. Exclusion Criteria. Exclusion criteria were determined according to previous reports [38-40]. The following exclusion criteria were used: (1) pregnancy and lactation; (2) renal failure with a creatinine level $>3 \mathrm{mg} / \mathrm{dL}$; (3) multiple myeloma; (4) history of hypersensitivity; (5) cardiogenic shock or left ventricular ejection fraction $<40 \%$; (6) patients undergoing heart transplants; (7) patients undergoing cardiac resynchronization therapy (CRT); (8) having implantable defibrillators (ICD); (9) difficult communication and other reasons.

2.4. Groups. Danshen compounds were extracted by ethanol and the quality was controlled according to the standard designed by China State Food and Drug Administration (http://www.sda.gov.cn/WS01/CL1236/114286.html). The main contents of ethanol extracts are tanshinone IIA, cryptotanshinone, tanshinone I [41], rosmarinic acid, and salvianolic acid B [42]. Danshen pills were the extracts of S. miltiorrhiza and provided as $27 \mathrm{mg} /$ pill by Tianjin Tasly Group Co., Ltd (Tianjin, China). Danshen pill is composed of $0.28 \%$ tanshinone IIA, $0.21 \%$ cryptotanshinone, $0.04 \%$ tanshinone I, 1.2\% rosmarinic acid, 5.8\% salvianolic acid B, and most starch. After the selection of inclusion and exclusion criteria, final 126 patients were evenly and randomly assigned into two groups: Danshen group and control group. Each person was assigned to one group using an electronic spreadsheet with the indicated number. To avoid the blinding of this study, three-month run-in period was added. During the period, all patients were treated as usual. Meanwhile, to keep the stable results, the changes of lifestyle and daily food calorie intake were discouraged. CHD patients in both groups had in-person visits or telephone contact in each week. The biochemical indices were maintained constant between two groups after 3-month run-in period and then entered treatment period with Danshen.

After three-month run-in period, the patients received 10 Danshen pills/time in Dashen group [43], while the control patients received placebo pills, three times daily. Meanwhile, all patients receive the normal therapy as in run-in period and the changes of lifestyle and daily food calorie intake were discouraged. CHD patients in both groups had in-person visits or telephone contact in each week. The duration of follow-up was 3 months.

2.5. The Measurement of Biochemical Indices. Blood sample was obtained from the antecubital vein of each patient on the day of enrollment, after 3-month run-in period, and 3-month administration of Danshen or placebo. Serum was separated from peripheral venous blood $(4 \mathrm{~mL})$ after centrifuge at $4^{\circ} \mathrm{C}$ at $3000 \mathrm{rpm}$ for $10 \mathrm{~min}$. The biochemical indices were measured, including lipid profiles (LDL-C, TC, TG, HDLC, ApoA, ApoB, ApoE, and Lp(a)), markers of liver function (GGT, TBil, IBil, and DBil), marker of renal function (UA), and a risk factor for cardiovascular disease (Hcy).

All kits were commercially available. Low-density lipoprotein cholesterol (LDL-C) BioAssay ELISA Kit (Human), Cat. number 196116, was from Beijing Huamei Scientific (Beijing, China). High-density lipoprotein cholesterol, HDLC, ELISA Kit, Cat. number CSB-E08954h, was from Cusabio 
Biotech Co., Ltd (Wuhan, China). Human total cholesterol (TC) ELISA Kit, Cat. number QY-E00062, was from Qayee Bio-Technology Co., Ltd (Shanghai, China). Human TG (Triglyceride) ELISA Kit, Cat. number E-EL-H5437, was from Elabscience Biotechnology Co., Ltd (Beijing, China). Lipoprotein A (ApoA) Human ELISA Kit, Cat. Number ab108878, Apolipoprotein B (ApoB) Human ELISA kit, Cat. number ab108807, and Apolipoprotein E (ApoE) Human ELISA Kit, Cat. number ab108813, were from Abcam Trading (Shanghai) Company, Ltd (Shanghai, China). ELISA Kit for Lipoprotein (a), Lp(a), Cat. number SEA842Hu, was from Wuhan USCN Business Co., Ltd (Wuhan, China). Human gamma-glutamyl transpeptidase, GGT ELISA Kit, Cat. number E1375h, was from Everlight Biotech (Taipei, Taiwan). Total Bilirubin, Human, ELISA Kit, Cat. number E01T0143, was from ARP American Research Products, Inc (Waltham, MA, USA). Bilirubin (Total and Direct) Colorimetric Assay Kit, Cat. number K553-100, was from BioVision, Inc (Milpitas, CA, USA). Uric Acid Assay Kit, Cat. number KA1651, was from Anova Corporation (Taipei, Taiwan). Human Homocysteine (HCY) ELISA Kit, Cat. number, was from Flarebio Biotech (Wuhan, China).

Just as in a run-in period, in order to avoid the variations in biochemical indices because of normal therapy, the changes of daily food calorie intake, and lifestyle, all these changes were discouraged in three-month treatment period. After three-month follow-up, serum biochemical indices were measured on all available data. These variables still include serum lipid profiles (LDL-C, HDL-C, TC, TG, ApoA, $\mathrm{ApoB}, \mathrm{ApoE}$, and $\mathrm{Lp}(\mathrm{a}))$; serum markers of liver function, GGT, TBil, IBil, and DBil; serum marker of renal function, $\mathrm{UA}$, and CHD risk factor, Hcy.

2.6. Statistical Analysis. A total of 126 patients (63 in each group) provided $90 \%$ power to detect the difference between two groups with an alpha level set at 0.05. All data were presented as mean values $\pm \mathrm{SD}$. Chi-squared test and $t$-test were applied. Analysis of variance was used to compare the serum levels of lipids at baseline and after 3-month treatment period in each group. $P<0.05$ (2-tailed) will be regarded as statistically significant. The analysis was conducted by using SPSS version 20.0 (IBM corporation; Chicago, IL, USA).

\section{Results}

3.1. Baseline Characters of CHD Patients. A total of 432 patients attended our hospital from March 2011 to June 2012. Of these patients, 306 CHD patients were excluded after selection with inclusion and exclusion criteria (Figure 1). Before administration of Danshen, 3-month run-in period was performed to make sure that there was no significant change in biochemical indices, although some of these patients are still taking the medicine for CHD therapy. Thus, 126 patients were selected and were randomly assigned to two groups: the Danshen group $(n=63)$ and the control group $(n=63)$. After another 3-month follow-up, 61 and 62 CHD patients finished the study in Danshen and control groups, respectively.
There was no significant difference for the clinical and procedural characteristics between Danshen and control groups (Table 1) $(P>0.05)$, including age, sex, risk element, clinical presentation, preprocedural laboratory results, and medication. There were $26(41.3 \%)$ and 24 (38.1\%) males in Danshen and control groups, respectively. The age of all CHD patients ranged from 60.2 to 73.5 years. Most $\mathrm{CHD}$ patients had unstable angina with 37 cases (58.7\%) in Danshen group and 34 cases (54.0\%) in control group. More than half number of patients was overweight according to BMI values (overweight $=$ BMI of 25-29.9) in both groups [44]. Hypertension was an obvious symptom with 48 cases $(76.2 \%)$ in Danshen group and 50 cases (79.4\%) in control group.

3.2. Biochemical Indices at Baseline. Serum biochemical indices were analyzed on all available data, to primarily identify these variables associated with $\mathrm{CHD}$ risk. These variables include serum lipid profiles (LDL-C, HDL-C, TC, TG, ApoA, ApoB, ApoE, and Lp(a)); serum markers of liver function, GGT, TBil, IBil, and DBil; serum marker of renal function, UA and CHD risk factor, and Hcy. All serum biochemical indices between Danshen and control groups were statistically insignificant $(P>0.05)$ (Table 2$)$.

3.3. Biochemical Indices after 3-Month Run-In Period. In order to avoid the variations in biochemical indices because of normal therapy, the changes of daily food calorie intake, and lifestyle, it is necessary to add three-month run-in period to make sure of the variations. Meanwhile, all these changes were discouraged. After three-month run-in period, serum biochemical indices were measured on all available data, which are associated with $\mathrm{CHD}$ risk. These variables still include serum lipid profiles (LDL-C, HDL-C, TC, TG, ApoA, ApoB, ApoE, and Lp(a)); serum markers of liver function, GGT, TBil, IBil, and DBil; serum marker of renal function, UA and CHD risk factor, and Hcy. The results also showed that there was no significantly statistical difference for these serum biochemical indices between Danshen and control groups after three-month run-in period (Table 3$)(P>0.05)$.

3.4. Analysis of Biochemical Indices after Three-Month Administration of Danshen. After three-month follow-up, two persons dropped out from Danshen group and one patient dropped out from control group. Thus, 61 and 62 patients finished the trial in Danshen and control groups (Figure 1), respectively. Danshen treatment reduced the levels of TG, TC, LDL-C, Lp(a), GGT, DBil, UA, and Hcy from median values (mg/dL) 114, 190, 113, 32, 3.3 (IU/dL), 0.4, 5.1, and 2.3 (Table 3 ) to media values (mg/dL) 101, 155, 98, 8, 1.6 (IU/dL), $0.2,4.5$, and 14 (Table 4$)$, respectively $(P<0.05)$. In contrast, Danshen treatment increased the levels of HDL-C, ApoA, ApoB, ApoE, TBil, and IBil from median values (mg/dL) 55, $98,76,7.0,0.5$, and 0.5 (Table 3 ) to median values $(\mathrm{mg} / \mathrm{dL}$ ) $62,119,93,8.7,0.8$, and 0.6 (Table 4 ), respectively $(P<0.05)$. Meanwhile, there were significantly statistical differences for these biochemical indices between Danshen and control groups after three-month follow-up (Table 4$)(P<0.05)$. Comparatively, there was no significantly statistical difference 


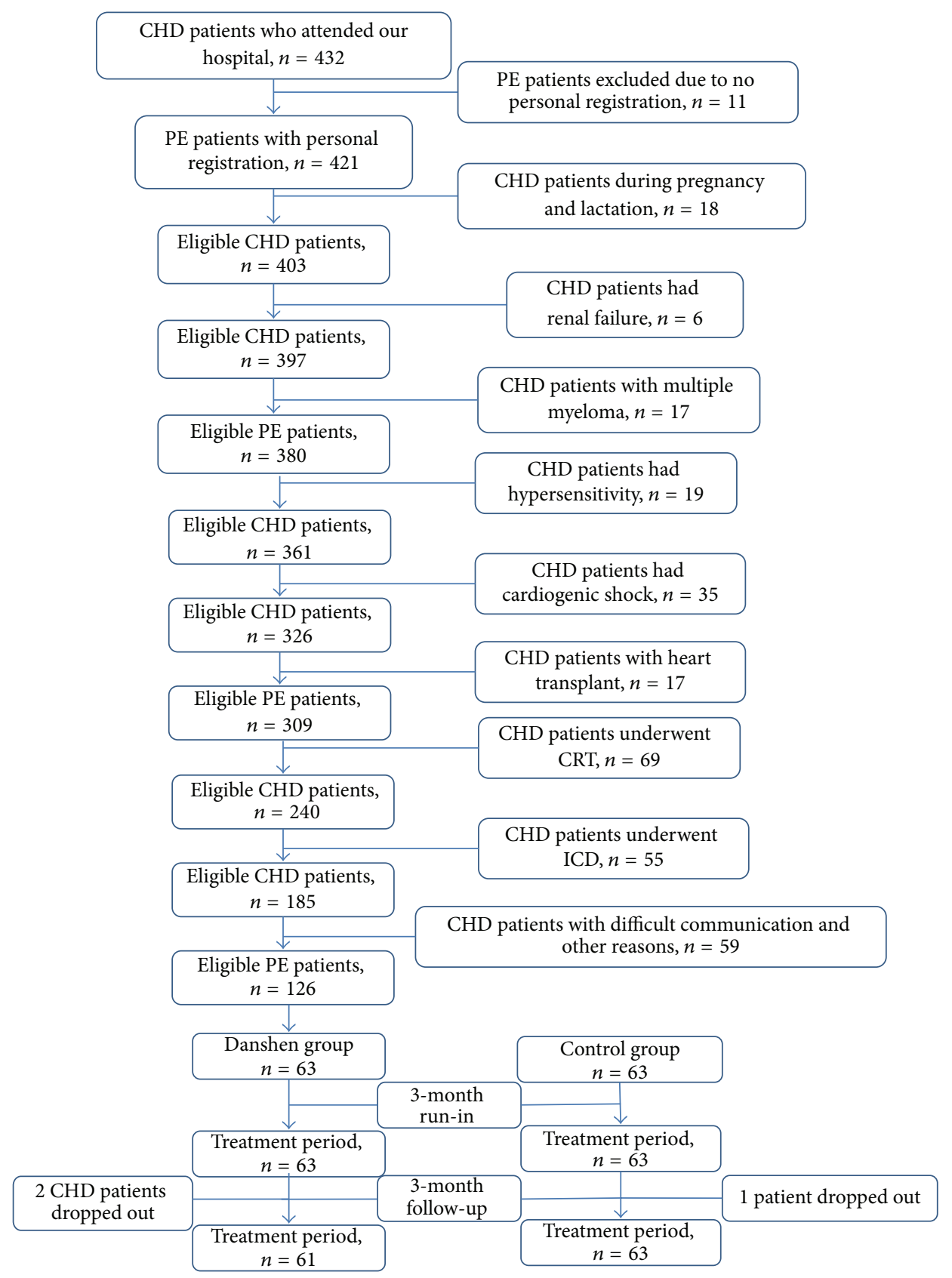

Figure 1: The flowchart of this study. CHD, coronary heart disease. The changes for CHD normal therapy, lifestyle, and daily food calories intake were discouraged in three-month run-in and three-month treatment periods. Finally, 61 and 62 CHD patients finished the whole procedure. Danshen pills were the extracts of Salvia miltiorrhiza and provided as $27 \mathrm{mg} /$ pill by Tianjin Tasly Group Co., Ltd (Tianjin, China).

for these biochemical parameters in control groups between run-in and treatment periods (Tables 3 and 4$)(P>0.05)$.

\section{Discussion}

The TCM Danshen has been long regarded as effective in "activating circulation and dispersing blood stasis" [45]. According to the classic theory of TCM, it has been said that "pain will be relieved when blockage is removed." The concept suggests that low blood circulation will do damage to human tissues and organs. Thus, such theory can be used for the management of CHD.

We assessed the therapeutic efficacy of Danshen, which is associated with the changes of lipid profiles in CHD patients. Multiple markers of biochemical indices of $\mathrm{CHD}$ patients were measured in the serum. The results indicated that Danshen presence may cause an improvement of several studied biochemical indices of $\mathrm{CHD}$ patients. Levels of TG, TC, LDL-C, Lp(a), GGT, DBil, the AU, and Hcy were statistically significantly reduced $(P<0.05$, resp.) 
TABLE 1: Baseline characters of CHD patients.

\begin{tabular}{|c|c|c|c|}
\hline Characteristic & Danshen $(n=63)$ & Control $(n=63)$ & $P$ values \\
\hline Age (years) & $65.9 \pm 5.7$ & $67.1 \pm 6.4$ & 0.269 \\
\hline Gender, male (\%) & $26(41.3)$ & $24(38.1)$ & 0.716 \\
\hline \multicolumn{4}{|l|}{ Hypertension } \\
\hline Systolic blood pressure $\geq 140 \mathrm{mmHg}$ or diastolic blood pressure $\geq 90 \mathrm{mmHg}(\%)$ & $48(76.2)$ & $50(79.4)$ & 0.668 \\
\hline Hypercholesterolemia (>200 mg/dL) (\%) & $21(33.3)$ & $22(34.9)$ & 0.851 \\
\hline Diabetes mellitus (\%) & $17(27)$ & $14(22.2)$ & 0.535 \\
\hline $\operatorname{BMI}\left(\mathrm{kg} / \mathrm{m}^{2}\right)$ & $25.2 \pm 5.6$ & $25.8 \pm 5.4$ & 0.541 \\
\hline Cigarette smokers (\%) & $31(49.2)$ & $29(46)$ & 0.721 \\
\hline Chronic kidney disease (\%) & $2(3.2)$ & $1(1.6)$ & 1.000 \\
\hline \multicolumn{4}{|l|}{ Clinical presentation } \\
\hline Unstable angina (\%) & $37(58.7)$ & $34(54)$ & 0.590 \\
\hline Non-ST-segment elevation myocardial infarction (\%) & $11(17.5)$ & $13(20.6)$ & 0.650 \\
\hline ST-segment elevation myocardial infarction (\%) & $12(19)$ & $14(22.2)$ & 0.660 \\
\hline
\end{tabular}

TABLE 2: Biochemical indices measure at baseline, median (range), mg/dL.

\begin{tabular}{|c|c|c|c|}
\hline & Danshen group $(n=63)$ & Placebo group $(n=63)$ & $P$ values \\
\hline \multicolumn{4}{|l|}{ Lipid profile } \\
\hline LDL cholesterol & $118(95-151)$ & $123(94-154)$ & 0.875 \\
\hline HDL cholesterol & $52(43-64)$ & $54(46-67)$ & 0.436 \\
\hline Total cholesterol & $197(178-239)$ & $191(172-243)$ & 0.527 \\
\hline Triglycerides & $117(85-168)$ & $121(84-171)$ & 0.329 \\
\hline Apolipoprotein A & $93(83-126)$ & $94(85-128)$ & 0.811 \\
\hline Apolipoprotein B & $71(62-104)$ & $73(65-99)$ & 0.743 \\
\hline Apolipoprotein E & $6.9(5.2-8.6)$ & $7.2(5.3-8.5)$ & 0.632 \\
\hline Lipoprotein (a) & $30(20-45)$ & $33(24-41)$ & 0.237 \\
\hline \multicolumn{4}{|l|}{ Liver function } \\
\hline Gamma-glutamyl transpeptidase (IU/dL) & $2.8(2.1-5.3)$ & $3.0(2.5-5.1)$ & 0.165 \\
\hline Total bilirubin & $0.5(0.3-0.6)$ & $0.6(0.4-0.8)$ & 0.175 \\
\hline Indirect bilirubin & $0.4(0.3-0.6)$ & $0.5(0.3-0.5)$ & 0.268 \\
\hline Direct bilirubin & $0.4(0.2-0.5)$ & $0.3(0.2-0.4)$ & 0.377 \\
\hline \multicolumn{4}{|l|}{ Renal function } \\
\hline Uric acid & $5.1(4.5-6.9)$ & $5.5(4.1-6.4)$ & 0.264 \\
\hline \multicolumn{4}{|l|}{ Risk factor of heart disease } \\
\hline Homocysteine & $22(19-40)$ & $23(21-42)$ & 0.459 \\
\hline
\end{tabular}

(Tables 3 and 4), while the levels of HDL-C, ApoA, ApoB, ApoE, TBIL, and IBil were significantly elevated (Tables 3 and $4)$, $(P<0.05$, resp. $)$. Most of these results are accordant with previous reports.

Danshen was able to improve liver function by increasing the level of total bilirubin [46] and reduce the level of UA in volunteers [47]. Hcy is a byproduct of methionine metabolism and its imbalance will result in hyperhomocysteinemia $[48,49]$, which is responsible for CHD development [50]. S. miltiorrhiza extract also inhibited unwanted adverse effects for HUVECs [51]. All these results suggest that the medicine can improve heart functions and is a potential drug in CHD therapy.

Danshen has been proved to suppress the intake of lowdensity lipoprotein, increase the expression of intercellular adhesion molecule, and modulate key events in atherosclerosis [52]. The combination of the medicine and Gegen can improve the ratios of TG/HDL-C and LDL-C/HDL-C [53]. The main compositions in Danshen may be beneficial to the improvement of lipid profiles. Cryptotanshinone showed protective effects on atherosclerosis of ApoE-deficient mice and can improve the situation caused by apolipoprotein shortage [54], which also was able to inhibit expression of oxidized adhesion molecules induced by LDL [55]. Tanshinone IIA showed scavenging effects on lipid free radicals in cardiac sarcoplasmic reticulum [56] and inhibited expression of oxidized low-density lipoprotein receptor-1 [57]. Ethanol extract of S. miltiorrhiza increased in vivo serum level of HDL to prevent the occurrence of osteoporosis [58]. Lipid peroxidation prevention was also observed in the case of its 
TABLE 3: Biochemical indices measure after three-month run-in period, median (range), mg/dL.

\begin{tabular}{|c|c|c|c|}
\hline & Danshen group $(n=63)$ & Placebo group $(n=63)$ & $P$ values \\
\hline \multicolumn{4}{|l|}{ Lipid profile } \\
\hline LDL cholesterol & $113(90-147)$ & $120(91-150)$ & 0.324 \\
\hline HDL cholesterol & $55(44-68)$ & $51(47-65)$ & 0.512 \\
\hline Total cholesterol & $190(172-234)$ & $189(176-248)$ & 0.763 \\
\hline Triglycerides & $114(87-173)$ & $118(82-166)$ & 0.262 \\
\hline Apolipoprotein A & $98(82-126)$ & $99(85-128)$ & 0.899 \\
\hline Apolipoprotein B & $76(66-103)$ & $78(62-109)$ & 0.842 \\
\hline Apolipoprotein E & $7.0(5.8-8.5)$ & $7.2(5.4-8.8)$ & 0.763 \\
\hline Lipoprotein (a) & $32(22-45)$ & $34(25-48)$ & 0.268 \\
\hline \multicolumn{4}{|l|}{ Liver function } \\
\hline Gamma-glutamyl transpeptidase (IU/dL) & $3.3(2.5-5.4)$ & $3.1(2.5-5.3)$ & 0.275 \\
\hline Total bilirubin & $0.5(0.4-0.7)$ & $0.6(0.4-0.8)$ & 0.431 \\
\hline Indirect bilirubin & $0.5(0.3-0.5)$ & $0.4(0.3-0.5)$ & 0.176 \\
\hline Direct bilirubin & $0.4(0.2-0.6)$ & $0.4(0.2-0.5)$ & 0.185 \\
\hline \multicolumn{4}{|l|}{ Renal function } \\
\hline Uric acid & $5.1(4.2-6.8)$ & $5.3(4.2-6.4)$ & 0.267 \\
\hline \multicolumn{4}{|l|}{ Risk factor of heart disease } \\
\hline Homocysteine & $23(20-43)$ & $25(20-46)$ & 0.341 \\
\hline
\end{tabular}

TABLE 4: Biochemical indices after three-month follow-up, median (range), mg/dL.

\begin{tabular}{|c|c|c|c|}
\hline & Danshen group $(n=61)$ & Placebo group $(n=62)$ & $P$ values \\
\hline \multicolumn{4}{|l|}{ Lipid profile } \\
\hline LDL cholesterol & $98(82-133)$ & $123(94-157)$ & 0.017 \\
\hline HDL cholesterol & $62(49-77)$ & $50(49-69)$ & 0.039 \\
\hline Total cholesterol & $155(147-195)$ & $192(179-251)$ & 0.001 \\
\hline Triglycerides & $101(80-158)$ & $121(84-172)$ & 0.016 \\
\hline Apolipoprotein A & $119(103-143)$ & $96(81-123)$ & 0.023 \\
\hline Apolipoprotein B & $93(78-114)$ & $75(65-101)$ & 0.009 \\
\hline Apolipoprotein E & $8.7(6.8-10.7)$ & $7.3(5.6-8.7)$ & 0.024 \\
\hline Lipoprotein (a) & $18(15-20)$ & $31(21-43)$ & 0.001 \\
\hline \multicolumn{4}{|l|}{ Liver function } \\
\hline Gamma-glutamyl transpeptidase (IU/dL) & $1.6(1.2-1.8)$ & $3.1(2.3-5.2)$ & 0.001 \\
\hline Total bilirubin & $0.8(0.6-1.0)$ & $0.6(0.4-0.7)$ & 0.032 \\
\hline Indirect bilirubin & $0.6(0.5-0.8)$ & $0.4(0.3-0.5)$ & 0.025 \\
\hline Direct bilirubin & $0.2(0.1-0.3)$ & $0.3(0.2-0.4)$ & 0.037 \\
\hline \multicolumn{4}{|l|}{ Renal function } \\
\hline Uric acid & $4.5(3.6-5.5)$ & $5.4(4.2-6.7)$ & 0.040 \\
\hline \multicolumn{4}{|l|}{ Risk factor of heart disease } \\
\hline Homocysteine & $14(11-17)$ & $24(20-41)$ & 0.001 \\
\hline
\end{tabular}

active compound-rosmarinic acid [59]. All these compositions may be beneficial to the improvement of lipid profiles of CHD patients.

The incidence of CHD differs widely among different studies. The determination of degree of correlation between the risk profiles and the prevalence of factors of CHD patients is often very complicated, especially in the patients with the cooccurring diseases such as hypertension [60, 61], diabetes mellitus [62, 63], renal disease [64], and others making such results more variable. Other activities also can make CHD become worse, such as oxidative stress $[65,66]$ and the production of proinflammatory cytokines [67]. Thus, the prevention of these accompanying diseases and these processes may improve the clinical outcome of CHD patients. More importantly, the progression of CHD by Danshen seems to be depended on its multiple functions and beneficial effects were demonstrated in several studies. For example, the medicine has the main components with antioxidant activities [10], which can prevent vascular injury [68]. It possesses anti-inflammatory properties [12], inhibits the aggregation of platelet [69], prevents thrombosis [70], reduces blood viscosity, and improves myocardial ischemia [71]. 
All these results suggest that Danshen is superior to most present medicine with multiple activities, which are beneficial to improve the symptoms of CHD. However, here, we only consider Danshen improving the lipid profiles of $\mathrm{CHD}$ patients. Much work needs to be done to better understand its function for ameliorating the severity of CHD.

It should be emphasized that the values of obtained results even with the clinical trial's limitation could have an impact; for example, (1) the sample size seems small only with 126 selected CHD patients, which is caused by the strict criteria given in this study; (2) the whole follow-up period is 6 months, while the period for administration of Danshen is only three months. In such short period, reduction of cardiac death and heart failure cannot be detected; (3) the safety of the medicine is not identified, although it has been widely used clinically in China. Our results should be counted as a promising, although preliminary. Much more evidence is needed to support the clinical use of Danshen for CHD patients.

\section{Conclusion}

Danshen was able to improve biochemical indices of $\mathrm{CHD}$ patients. In a prespecified exploratory analysis, there was evidence of a reduction in the rate of $\mathrm{CHD}$ events among patients who had received the medicine therapy. Presently, it is the most popular Chinese herbal drug and is often used either alone or in combination with other drugs, especially for the therapies of cardiovascular diseases. Results of our study reflect the global trend of studies in the field of the role of Danshen in therapy development for CHD patients.

\section{Competing Interests}

The authors declare that there are no competing interests regarding the publication of this paper.

\section{Acknowledgments}

The authors thank Dr. Wang for her technical assistance during data collection.

\section{References}

[1] J. A. Leigh, M. Alvarez, and C. J. Rodriguez, "Ethnic minorities and coronary heart disease: an update and future directions," Current Atherosclerosis Reports, vol. 18, no. 2, article 9, 2016.

[2] J. S. Kanu, Y. Gu, S. Zhi et al., "Single nucleotide polymorphism rs3774261 in the AdipoQ gene is associated with the risk of coronary heart disease (CHD) in Northeast Han Chinese population: a case-control study," Lipids in Health and Disease, vol. 15, no. 1, p. 6, 2016.

[3] L. Anderson, D. R. Thompson, N. Oldridge et al., "Exercisebased cardiac rehabilitation for coronary heart disease," Journal of the American College of Cardiology, vol. 67, no. 1, pp. 1-12, 2016.

[4] X.-Y. Zhang, L. Shu, C.-J. Si et al., "Dietary patterns, alcohol consumption and risk of coronary heart disease in adults: a meta-analysis," Nutrients, vol. 7, no. 8, pp. 6582-6605, 2015.
[5] S. Zhao, H. Zhao, L. Wang, S. Du, and Y. Qin, "Education is critical for medication adherence in patients with coronary heart disease," Acta Cardiologica, vol. 70, no. 2, pp. 197-204, 2015.

[6] S. K. Agarwal, A. Kapoor, S. Pande et al., "Comparison of release kinetics of different cardiac biomarkers in patients undergoing off pump coronary artery bypass surgery and valve replacement surgery for rheumatic heart disease," Journal of Cardiothoracic Surgery, vol. 10, supplement 1, article A208, 2015.

[7] H. Cao, J. Zhai, W. Mu et al., "Use of comparative effectiveness research for similar Chinese patent medicine for angina pectoris of coronary heart disease: a new approach based on patientimportant outcomes," Trials, vol. 15, article 84, 2014.

[8] J. Luo, W. Song, G. Yang, H. Xu, and K. Chen, "Compound Danshen (Salvia miltiorrhiza) dripping pill for coronary heart disease: an overview of systematic reviews," The American Journal of Chinese Medicine, vol. 43, no. 1, pp. 25-43, 2015.

[9] T. O. Cheng, "Danshen: a versatile Chinese herbal drug for the treatment of coronary heart disease," International Journal of Cardiology, vol. 113, no. 3, pp. 437-438, 2006.

[10] G.-J. Zhou, W. Wang, X.-M. Xie, M.-J. Qin, B.-K. Kuai, and T.S. Zhou, "Post-harvest induced production of salvianolic acids and significant promotion of antioxidant properties in roots of Salvia miltiorrhiza (Danshen)," Molecules, vol. 19, no. 6, pp. 7207-7222, 2014.

[11] J.-Y. Han, J.-Y. Fan, Y. Horie et al., "Ameliorating effects of compounds derived from Salvia miltiorrhiza root extract on microcirculatory disturbance and target organ injury by ischemia and reperfusion," Pharmacology and Therapeutics, vol. 117, no. 2, pp. 280-295, 2008.

[12] C. Stumpf, Q. Fan, C. Hintermann et al., "Anti-inflammatory effects of Danshen on human vascular endothelial cells in culture," The American Journal of Chinese Medicine, vol. 41, no. 5, pp. 1065-1077, 2013.

[13] C. Wang, R. Zhao, B. Li, L. Y. Gu, and H. Gou, "An in vivo and in vitro study: high-dosage Danshen injection induces peripheral vascular endothelial cells injury," Human \& Experimental Toxicology, vol. 35, no. 4, pp. 404-417, 2016.

[14] Y. Chen, N. Zhang, J. Ma et al., "A Platelet/CMC coupled with offline UPLC-QTOF-MS/MS for screening antiplatelet activity components from aqueous extract of Danshen," Journal of Pharmaceutical and Biomedical Analysis, vol. 117, pp. 178-183, 2016.

[15] C. M. Gamboa, M. M. Safford, E. B. Levitan et al., "Statin underuse and low prevalence of LDL-C control among U.S. adults at high risk of coronary heart disease," American Journal of the Medical Sciences, vol. 348, no. 2, pp. 108-114, 2014.

[16] Y. Huang, H. D. Ye, X. Gao et al., "Significant interaction of APOE rs4420638 polymorphism with HDL-C and APOA-I levels in coronary heart disease in Han Chinese men," Genetics and Molecular Research, vol. 14, no. 4, pp. 13414-13424, 2015.

[17] P. Gong, S. Li, L. Hu et al., "Total cholesterol mediates the effect of ABO blood group on coronary heart disease," Zhonghua Xin Xue Guan Bing Za Zhi, vol. 43, no. 5, pp. 404-407, 2015.

[18] A. Onat, "Influence of gender, C- reactive protein and triglycerides in risk prediction of coronary heart disease," Anadolu Kardiyoloji Dergisi, vol. 13, no. 3, pp. 287-288, 2013.

[19] L. Zhu, Z. Lu, and Y. Y. Song, "Advances in the association between apolipoprotein (a) gene polymorphisms and coronary heart disease," Zhongguo Yi Xue Ke Xue Yuan Xue Bao, vol. 37, no. 4, pp. 482-488, 2015. 
[20] J. Z. Zhang, Y. Y. Zheng, Y. N. Yang et al., "Association between apolipoprotein $\mathrm{B}$ gene polymorphisms and the risk of coronary heart disease (CHD): an update meta-analysis," Journal of the Renin-Angiotensin-Aldosterone System, vol. 16, no. 4, pp. 827837, 2015.

[21] G. D. Kolovou, V. Kolovou, D. B. Panagiotakos et al., "Study of common variants of the apolipoprotein $\mathrm{E}$ and lipoprotein lipase genes in patients with coronary heart disease and variable body mass index," Hormones, vol. 14, no. 3, pp. 376-382, 2015.

[22] E. V. Mazdorova, K. Y. Nikolaev, Y. V. Polonskaya et al., "Valuing the lipid marker of lipoprotein (a) in the diagnosis of patients with coronary heart disease," Kardiologiia, vol. 55, no. 8, p. 49, 2015.

[23] Y. Hashimoto, A. Futamura, H. Nakarai, and K. Nakahara, "Relationship between response of $\gamma$-glutamyl transpeptidase to alcohol drinking and risk factors for coronary heart disease," Atherosclerosis, vol. 158, no. 2, pp. 465-470, 2001.

[24] E. Oda and R. Kawai, "A possible cross-sectional association of serum total bilirubin with coronary heart disease and stroke in a Japanese health screening population," Heart and Vessels, vol. 27, no. 1, pp. 29-36, 2012.

[25] C. Ghem, R. E. Sarmento-Leite, A. S. de Quadros, S. Rossetto, and C. A. M. Gottschall, "Serum bilirubin concentration in patients with an established coronary artery disease," International Heart Journal, vol. 51, no. 2, pp. 86-91, 2010.

[26] F. Lin, H. Zhang, F. Huang, H. Chen, C. Lin, and P. Zhu, "Influence of changes in serum uric acid levels on renal function in elderly patients with hypertension: a retrospective cohort study with 3.5-year follow-up," BMC Geriatrics, vol. 16, no. 1, article 35, 2016.

[27] D. R. Kannangara, G. G. Graham, K. M. Williams, and R. O. Day, "Effect of xanthine oxidase inhibitors on the renal clearance of uric acid and creatinine," Clinical Rheumatology, 2016.

[28] B. Ekici, U. Kütük, A. Alhan, and H. F. Töre, “The relationship between serum uric acid levels and angiographic severity of coronary heart disease," Kardiologia Polska, vol. 73, no. 7, pp. 533-538, 2015.

[29] L. Han, Q. Wu, C. Wang et al., "Homocysteine, ischemic stroke, and coronary heart disease in hypertensive patients: a population-based, prospective cohort study," Stroke, vol. 46, no. 7, pp. 1777-1786, 2015.

[30] E. Sertoglu, H. Kayadibi, and M. Uyanik, "Biochemical view on 'Homocysteine and metabolic syndrome: from clustering to additional utility in prediction of coronary heart disease," Journal of Cardiology, vol. 65, no. 5, p. 439, 2015.

[31] Z. Li, L. Zhu, and B. Huang, "Effects of purified herbal extract of Salvia miltiorrhiza on lipid profile in hyperlipidemic patients," Journal of Geriatric Cardiology, vol. 6, no. 2, pp. 99-101, 2009.

[32] C. Zhu, H. Cao, X. Zhou et al., "Meta-analysis of the clinical value of danshen injection and huangqi injection in liver cirrhosis," Evidence-Based Complementary and Alternative Medicine, vol. 2013, Article ID 842824, 8 pages, 2013.

[33] X. Lu, Y. Jin, L. Ma, and L. Du, "Danshen (Radix Salviae Miltiorrhizae) reverses renal injury induced by myocardial infarction," Journal of Traditional Chinese Medicine, vol. 35, no. 3, pp. 306-311, 2015.

[34] General Assembly of the World Medical Association, "World Medical Association Declaration of Helsinki: ethical principles for medical research involving human subjects," The Journal of the American College of Dentists, vol. 81, no. 3, pp. 14-18, 2014.
[35] American College of Cardiology and American Heart Association Task Force on Practice, "ACC/AHA guidelines for the management of patients with unstable angina and non-ST segment elevation myocardial infarction: executive summary and recommendations," Catheterization and Cardiovascular Interventions, vol. 51, no. 4, pp. 505-521, 2000.

[36] S. Senol, M. U. Es, G. Gokmen, O. Ercin, B. A. Tuylu, and K. Kargun, "Genetic polymorphisms in preoperative myocardial infarction," Asian Cardiovascular and Thoracic Annals, vol. 23, no. 4, pp. 389-393, 2015.

[37] T. N. Sheth, O. A. Kajander, S. Lavi et al., "Optical coherence tomography-guided percutaneous coronary intervention in ST-segment-elevation myocardial infarction: a prospective propensity-matched cohort of the thrombectomy versus percutaneous coronary intervention alone trial," Circulation: Cardiovascular Interventions, vol. 9, no. 4, Article ID e003414, 2016.

[38] M. A. Becker, H. R. Schumacher Jr., R. L. Wortmann et al., "Febuxostat, a novel nonpurine selective inhibitor of xanthine oxidase: a twenty-eight-day, multicenter, phase II, randomized, double-blind, placebo-controlled, dose-response clinical trial examining safety and efficacy in patients with gout," Arthritis \& Rheumatism, vol. 52, no. 3, pp. 916-923, 2005.

[39] S. H. Park, M. H. Jeong, I. H. Park et al., "Effects of combination therapy of statin and $\mathrm{N}$-acetylcysteine for the prevention of contrast-induced nephropathy in patients with ST-segment elevation myocardial infarction undergoing primary percutaneous coronary intervention," International Journal of Cardiology, vol. 212, pp. 100-106, 2016.

[40] A. Auricchio, C. Stellbrink, S. Sack et al., "Long-term clinical effect of hemodynamically optimized cardiac resynchronization therapy in patients with heart failure and ventricular conduction delay," Journal of the American College of Cardiology, vol. 39, no. 12, pp. 2026-2033, 2002.

[41] F. Qiu, J. Jiang, Y. Ma et al., “Opposite effects of single-dose and multidose administration of the ethanol extract of danshen on CYP3A in healthy volunteers," Evidence-Based Complementary and Alternative Medicine, vol. 2013, Article ID 730734, 8 pages, 2013.

[42] X. Gong, Y. Li, and H. Qu, "Removing tannins from medicinal plant extracts using an alkaline ethanol precipitation process: a case study of Danshen injection," Molecules, vol. 19, no. 11, pp. 18705-18720, 2014.

[43] Y. Jia, F. Huang, S. Zhang, and S.-W. Leung, "Is danshen (Salvia miltiorrhiza) dripping pill more effective than isosorbide dinitrate in treating angina pectoris? A systematic review of randomized controlled trials," International Journal of Cardiology, vol. 157, no. 3, pp. 330-340, 2012.

[44] C. L. Ogden and M. D. Carroll, Prevalence of Overweight, Obesity, and Extreme Obesity among Adults: United States, Trends 1960-1962 through 2007-2008, vol. 6, National Center for Health Statistics, Hyattsville, Md, USA, 2010.

[45] T. O. Cheng, "Cardiovascular effects of Danshen," International Journal of Cardiology, vol. 121, no. 1, pp. 9-22, 2007.

[46] G.-I. Zhang, X.-D. Wei, B.-J. Fang et al., "The study of preventing hepatic veno-occlusive disease with danshen for injection," Proceeding of Clinical Medicine, vol. 8, article 30, 2010.

[47] H. Wu and X. Zhang, "The study on the effect of compound danshen dripping pills on the activity of CYP1A2, NAT2, and XO," Chinese Journal of Modern Applied Pharmacy, vol. 1, article 5, 2009.

[48] A. H. Hainsworth, N. E. Yeo, E. M. Weekman, and D. M. Wilcock, "Homocysteine, hyperhomocysteinemia and vascular 
contributions to cognitive impairment and dementia (VCID)," Biochimica et Biophysica Acta, vol. 1862, no. 5, pp. 1008-1017, 2016.

[49] E. Bukharaeva, A. Shakirzyanova, V. Khuzakhmetova, G. Sitdikova, and R. Giniatullin, "Homocysteine aggravates ROSinduced depression of transmitter release from motor nerve terminals: potential mechanism of peripheral impairment in motor neuron diseases associated with hyperhomocysteinemia," Frontiers in Cellular Neuroscience, vol. 9, article 391, 2015.

[50] C. Liu, Y. Yang, D. Peng, L. Chen, and J. Luo, "Hyperhomocysteinemia as a metabolic disorder parameter is independently associated with the severity of coronary heart disease," Saudi Medical Journal, vol. 36, no. 7, pp. 839-846, 2015.

[51] K. Chan, S. H. Chui, D. Y. L. Wong, W. Y. Ha, C. L. Chan, and R. N. S. Wong, "Protective effects of Danshensu from the aqueous extract of Salvia miltiorrhiza (Danshen) against homocysteineinduced endothelial dysfunction," Life Sciences, vol. 75, no. 26, pp. 3157-3171, 2004.

[52] D. P. Sieveking, K.-S. Woo, K. P. Fung, P. Lundman, S. Nakhla, and D. S. Celermajer, "Chinese herbs Danshen and Gegen modulate key early atherogenic events in vitro," International Journal of Cardiology, vol. 105, no. 1, pp. 40-45, 2005.

[53] J. Li, X. Cheng, J. Gu et al., "The effects of Gegen-Danshen prescription on the lipid metabolism in hyperlipidemia rats," Journal of Southwest University for Nationalities: Natural Science Edition, vol. 36, no. 6, pp. 926-924, 2012.

[54] Z. Liu, S. Xu, X. Huang et al., "Cryptotanshinone, an orally bioactive herbal compound from Danshen, attenuates atherosclerosis in apolipoprotein E-deficient mice: role of lectin-like oxidized LDL receptor-1 (LOX-1)," British Journal of Pharmacology, vol. 172, no. 23, pp. 5661-5675, 2015.

[55] W. Zhao, C. Wu, and X. Chen, "Cryptotanshinone inhibits oxidized LDL-induced adhesion molecule expression via ROS dependent NF- $\kappa$ B pathways," Cell Adhesion \& Migration, 2015.

[56] W. Jiang, Y. Zhao, B. Zhao, Q. Wan, and W. Xin, "Studies on the scavenging effect of Tanshinone on lipid free radical of cardiac sarcoplasmic reticulum during peroxidation," Shengwu Wuli Xuebao, vol. 10, no. 4, pp. 685-689, 1993.

[57] S. Xu, Z. Liu, Y. Huang et al., "Tanshinone II-A inhibits oxidized LDL-induced LOX-1 expression in macrophages by reducing intracellular superoxide radical generation and NF- $\kappa \mathrm{B}$ activation," Translational Research, vol. 160, no. 2, pp. 114-124, 2012.

[58] Z.-P. Zhang, T.-T. You, L.-Y. Zou, T. Wu, Y. Wu, and L. Cui, "Effect of Danshen root compound on blood lipid and bone biomechanics in mice with hyperlipemia-induced osteoporosis," Nan Fang Yi Ke Da Xue Xue Bao, vol. 28, no. 9, pp. 15501553, 2008.

[59] O. Fadel, K. El Kirat, and S. Morandat, "The natural antioxidant rosmarinic acid spontaneously penetrates membranes to inhibit lipid peroxidation in situ," Biochimica et Biophysica Acta (BBA)_Biomembranes, vol. 1808, no. 12, pp. 2973-2980, 2011.

[60] E. D. Michos and P. L. Lutsey, "25-hydroxyvitamin D levels and coronary heart disease risk reclassification in hypertension-is it worth the 'hype'?" Atherosclerosis, vol. 245, pp. 237-239, 2016.

[61] A. A. Nargesi, B. Heidari, S. Esteghamati et al., "Contribution of vitamin D deficiency to the risk of coronary heart disease in subjects with essential hypertension," Atherosclerosis, vol. 244, pp. 165-171, 2016.

[62] J. Liu, Y. Zou, Y. Tang et al., "Circulating cell-free mitochondrial deoxyribonucleic acid is increased in coronary heart disease patients with diabetes mellitus," Journal of Diabetes Investigation, vol. 7, no. 1, pp. 109-114, 2016.

[63] R. N. Das, "Relationship between diabetes mellitus and coronary heart disease," Current Diabetes Reviews, vol. 12, no. 999, pp. 1-12, 2016.

[64] H. J. Dong, C. Huang, D. M. Luo et al., "Concomitant coronary and renal revascularization improves left ventricular hypertrophy more than coronary stenting alone in patients with ischemic heart and renal disease," Journal of Zhejiang University SCIENCE B, vol. 17, no. 1, pp. 67-75, 2016.

[65] N. Katakami, H. Kaneto, T.-A. Matsuoka et al., "Accumulation of oxidative stress-related gene polymorphisms and the risk of coronary heart disease events in patients with type 2 diabetesan 8-year prospective study," Atherosclerosis, vol. 235, no. 2, pp. 408-414, 2015.

[66] I. V. Gorudko, V. A. Kostevich, A. V. Sokolov et al., "Functional activity of neutrophils in diabetes mellitus and coronary heart disease: role of myeloperoxidase in the development of oxidative stress," Bulletin of Experimental Biology and Medicine, vol. 154, no. 1, pp. 23-26, 2012.

[67] V. M. Provotorov, A. V. Budnevskii, G. G. Semenkova, and E. S. Shishkina, "Proinflammatory cytokines in combination of coronary heart disease and chronic obstructive pulmonary disease," Klinicheskaia Meditsina, vol. 93, no. 2, pp. 5-9, 2015.

[68] G. L. Oktar, M. Kirisci, A. D. Dursun et al., "Antioxidative effects of adrenomedullin and vascular endothelial growth factor on lung injury induced by skeletal muscle ischemia-reperfusion," Bratislava Medical Journal, vol. 114, no. 11, pp. 625-628, 2013.

[69] S. T. Ma, G. L. Dai, X. G. Cheng et al., "Synergistic action of Compound Danshen Dripping Pill (CDDP) on Clopidogrel Bisulfate (CPG) counteracting platelet aggregation," Zhong Yao Cai, vol. 37, no. 10, pp. 1820-1825, 2014.

[70] Z. S. Huang, C. L. Zeng, L. J. Zhu, L. Jiang, N. Li, and H. $\mathrm{Hu}$, "Salvianolic acid A inhibits platelet activation and arterial thrombosis via inhibition of phosphoinositide 3-kinase," Journal of Thrombosis and Haemostasis, vol. 8, no. 6, pp. 1383-1393, 2010.

[71] T. Y. K. Chan, "Interaction between warfarin and danshen (Salvia miltiorrhiza)," Annals of Pharmacotherapy, vol. 35, no. 4, pp. 501-504, 2001. 


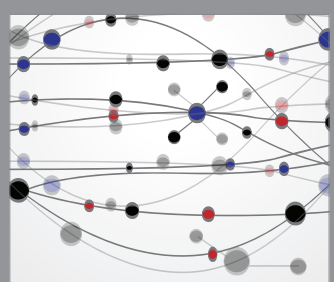

The Scientific World Journal
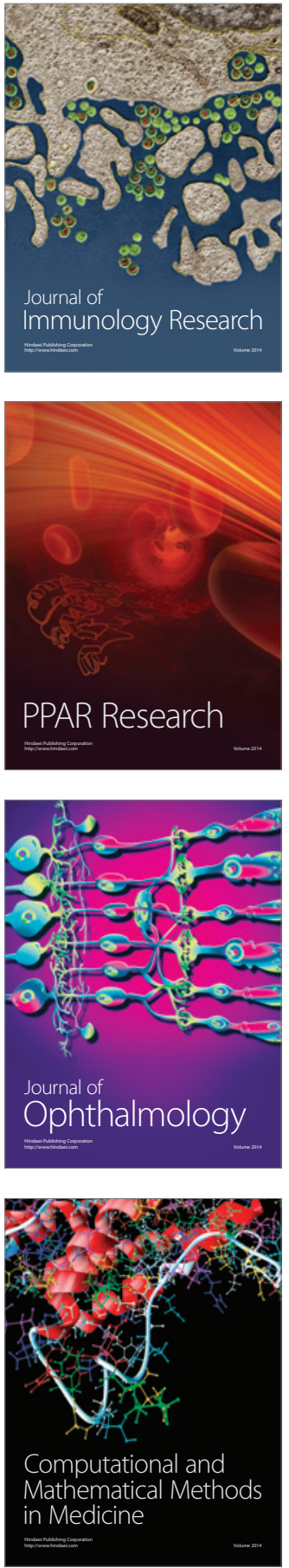

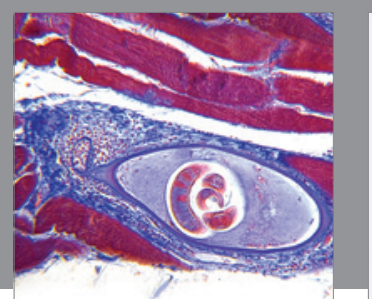

Gastroenterology Research and Practice

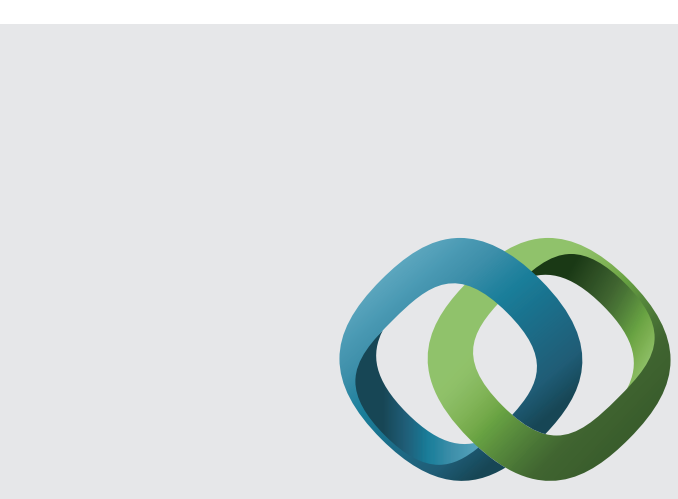

\section{Hindawi}

Submit your manuscripts at

http://www.hindawi.com
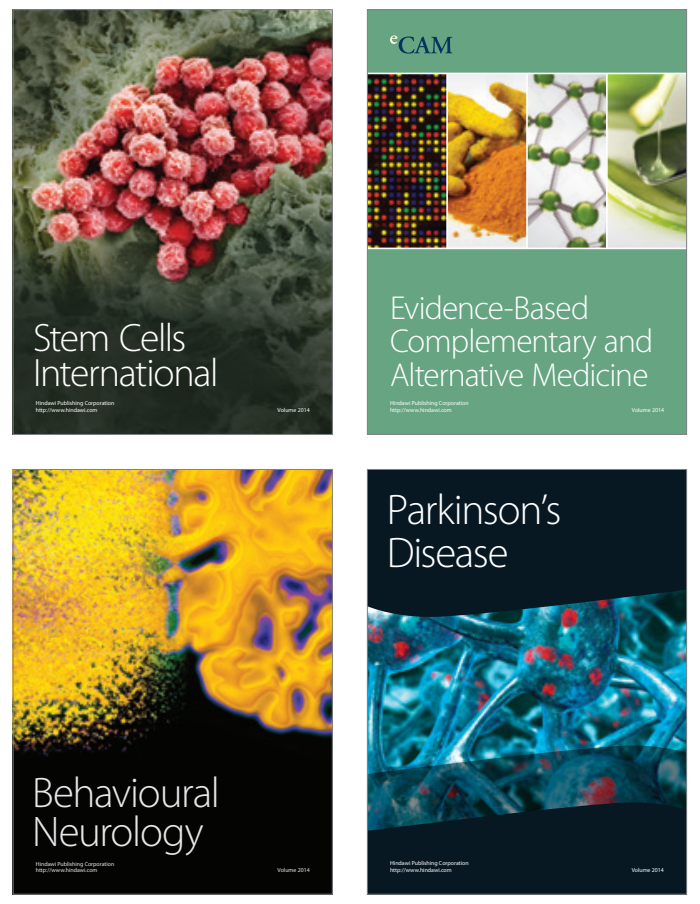
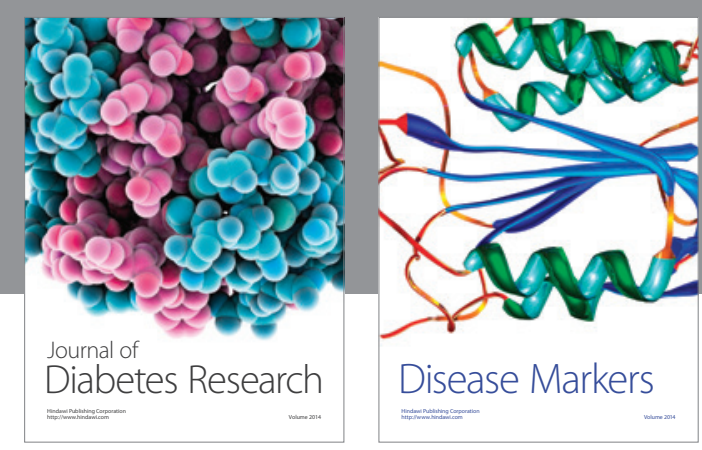

Disease Markers
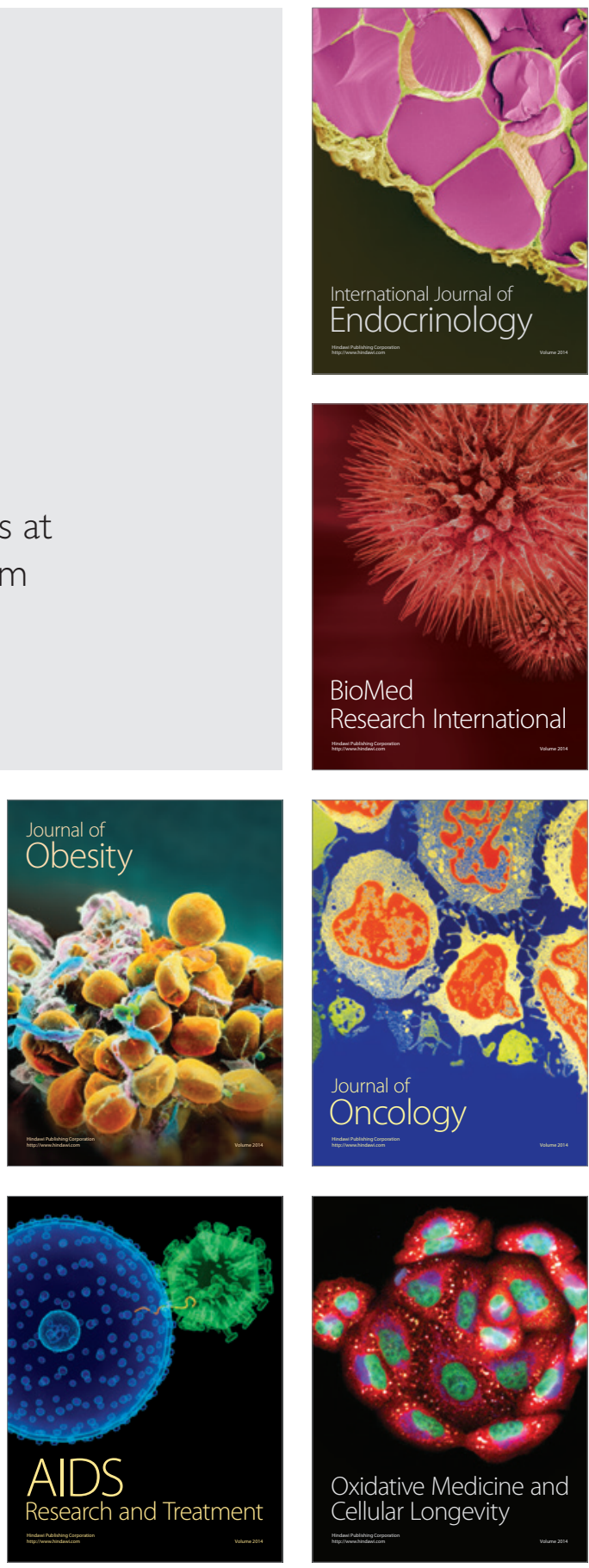